\title{
Phenotypic and Molecular Characterization of Carbapenamase among Acinetobacter Isolates in a Tertiary Care Hospital, Tamil Nadu, India
}

\author{
J. Thiriveni ${ }^{{ }^{*}}$, U. Umadevi ${ }^{2}$ and N. Lakshmipriya ${ }^{2}$ \\ ${ }^{1}$ Coimbatore Medical College, Coimbatore - 641 014, Tamil Nadu, India \\ ${ }^{2}$ Institute of Microbiology, Madras Medical College, Chennai- 600 003, Tamil Nadu, India \\ *Corresponding author
}

\section{Keywords}

MDR- Multi drug resistant, XDR- Extended drug resistant, PDR- Pan drug resistant, ESBLExtended spectrum beta lactamase, MHT-

Modified Hodge test, IEDT- Imipenem EDTA combined disc test

\section{Article Info}

Accepted:

20 November 2018

Available Online:

10 December 2018

\section{A B S T R A C T}

Acinetobacter the aerobic non fermenting GNCB are emerging as important pathogens in health care associated infection, exhibiting increased antimicrobial resistance by virtue of multiple resistant mechanisms with practically no therapeutic options. The objective was to determine the multitude of resistant mechanisms by phenotypic and genotypic characterization and the prevalent antimicrobial susceptibility pattern. 175 clinically significant non-duplicate Acinetobacter isolates were included. ESBL, AmpC and carbapenamase production were determined by CLSI phenotypic confirmatory method, AmpC disc test, Modified Hodge test and Imipenem-EDTA combined disc test respectively. Presence of genes conferring carbapenem resistance viz OXA23, blaVIM1 \& blaIMP1 were determined by PCR. A. baumannii $(81.14 \%)$ was the most common species with significant difference $(\mathrm{p}$ value $<0.05)$ in antimicrobial sensitivity pattern. MDR was $60 \%$, XDR was $11.43 \%$ and no PDR isolate. Carbapenem resistance was detected in 20 isolates $(11.43 \%)$. MIC of meropenem for the resistant isolates were between $32 \mu \mathrm{g} / \mathrm{ml}$ and $256 \mu \mathrm{g} / \mathrm{ml}$. MHT was positive in $9(45 \%)$ isolates and IEDT was positive in $9(45 \%)$ isolates. $61(34.86 \%)$ isolates were ESBL producers and 23(13.14\%) isolates were AmpC producers. On molecular characterization OXA-23 gene was detected in all 20 meropenem resistant isolates $(100 \%)$. MBL conferred by blaVIM1 gene was positive in 9 isolates (45\%) and blaIMP1 in 7 isolates (35\%). Even though genotypic methods are the gold standard, phenotypic methods due to ease of performance and cost effectiveness still remain useful for characterization of carbapenemase resistance.

\section{Introduction}

The Genus Acinetobacter because of its ubiquitous existence causes a wide range of clinical infections due to colonization and persistent infection resulting in high mortality among patients in ICU and immune compromised. The treatment of Acinetobacter infections remains a great challenge because of its different types of resistant mechanisms including ESBL, AmpC, Non-metallo beta lactamases and Metallo betalactamases, the most common emerging cause for antimicrobial resistance. ${ }^{(1),(2)}$ Carbapenems 
remains the drug of choice but resistance to carbapenems by the production of carbapenamases and various other mechanisms has limited the therapeutic options. $^{(3),(4)}$

Carbapenem resistance in Acinetobacter may be due to oxacillinases, metallo beta lactamases, AmpC beta lactamases or due to porin deficiency. ${ }^{(5)}$ Since oxacillinases are chromosomally mediated, spread of OXA genes to other organisms is less frequent when compared to MBL, where the spread is plasmid mediated and hence the propensity of dissemination is multifold. Also metallo beta lactamases are more potent (100-1000 fold) hydrolyzers of carbapenems when compared to OXA type carbapenamases which contribute to the carbapenem resistance to a greater extent. ${ }^{(6)}$ Hence this study is focused on identifying the carbapenamases among Acinetobacter isolates by phenotypic and genotypic methods.

\section{Materials and Methods}

This cross sectional study was conducted in 2700 bedded tertiary care referral hospital including the clinical specimens obtained from patients admitted in various clinical departments during the period of one year from Sep 2013 to Aug 2014. Approval from the Institutional ethics committee and informed consent from the patients in the study group were obtained.

A total of 175 clinically significant, consecutive, non duplicate isolates of Acinetobacter species from various clinical specimens blood, sputum, endo tracheal aspirate, bronchial wash, pleural fluid, ascitic fluid, peritoneal dialysis fluid, cerebrospinal fluid, urine and wound swabs were included and were processed at the clinical microbiology laboratory for phenotypic identification and antibiotic susceptibility testing as per standard protocol. The panel of antibiotics included was cefotaxime, ceftazidime, cefepime, amikacin, gentamycin, ciprofloxacin, cotrimoxazole, piperacillin tazobactum, imipenem, meropenem, polymyxin B and tigecycline.

All the isolates along with positive and negative control were subjected to Carbapenemase screening test using meropenem disc $10 \mu \mathrm{g}$ (Himedia). The isolates which were resistant to meropenem by disc diffusion method as indicated by a zone diameter $<18 \mathrm{~mm}$ as per CLSI guidelines were interpreted as carbapenamase producers and further tested for Oxacillinase, Metallo beta lactamase and AmpC beta lactamase production. ${ }^{(7),(8)}$

MIC for meropenem by Macro broth dilution method were determined for those isolates which were tested positive for carbapenemase production by screening test. ATCC Pseudomonas aeruginosa was used as control strain. The lowest concentration of the antibiotic which shows clearing was considered as MIC. Interpretation was done as follows $\leq 2 \mu \mathrm{g} / \mathrm{ml}$ as sensitive, $4 \mu \mathrm{g} / \mathrm{ml}$ as intermediate and $\geq 8 \mu \mathrm{g} / \mathrm{ml}$ as resistant.

\section{Oxacillinase detection by modified Hodge test}

MHT for detection of carbapenamase was performed as per Lee et al., ${ }^{(9)}$ A 0.5 McFarland standard suspension of E. coli ATCC 25922 from an overnight culture was prepared and was diluted 1:10 in saline or broth. The MHA plate was inoculated with the suspension as for disc diffusion procedure and allowed to dry for 5 to 10 minutes. The $10 \mu \mathrm{g}$ of Ertapenem disc (Himedia) was placed in the centre. The test isolate was then streaked with a length of 20 to $25 \mathrm{~mm}$ from the edge of the disc to the periphery of the petridish along with positive (Klebsiella pneumoniae ATCC 
BAA-1705) and negative control (Klebsiella pneumoniae ATCC BAA-1706) and incubated at $37^{\circ} \mathrm{C}$ for 24 hours. ${ }^{(10),(11)}$ Enhanced growth of the test strain towards the zone of inhibition was identified as positive for carbapenamase production. Similarly, no enhanced growth of the test strain towards the zone of inhibition as negative.

\section{Metallo Beta lactamase Detection by Imipenem (IMP)-EDTA Combined Disc Test}

The test isolates were inoculated onto MHA plates as for disc diffusion procedure. Two Imipenem discs $(10 \mu \mathrm{g})$ (Himedia) were placed on the MHA plate. $10 \mu$ of EDTA solution $(750 \mu \mathrm{g})$ was added to one of the Imipenem disc. The plate was incubated at $37^{\circ} \mathrm{C}$ for 24hrs. The increase in zone size of $\geq 7 \mathrm{~mm}$ around Imipenem EDTA as compared to Imipenem was interpreted as a positive result. $^{(9),(10),(12)}$

\section{AmpC Betalactamase Detection by AmpC Disc Test}

A lawn culture of ATCC E. coli 25922 was prepared on a MHA plate. A disc containing $10 \mu \mathrm{g}$ of cefoxitin (Himedia) was placed on the surface of agar. Sterile disc was moistened with $20 \mu$ sterile saline and inoculated with several colonies of the test organism. The inoculated disc was then placed beside the cefoxitin disc almost touching on the inoculated plate. The plates were incubated overnight at $37^{\circ} \mathrm{C}$. A positive test appears as flattening or indentation of the cefoxitin inhibition zone in the vicinity of the test disc. $^{(11),(13),(14)}$

\section{Molecular method (Polymerase chain reaction)}

All the isolates that were detected to be carbapenamase producers by the screening test were subjected to conventional PCR for the detection of oxacillinase gene OXA-23 and Metallo Beta Lactamases genes bla-IMP1 and bla-VIM1. ${ }^{(15),(16)}$ The primers used were shown in Table 1. The amplified PCR products were seen as bright fluorescent bands. Controls were included in each test run with satisfactory results.

\section{Statistical analysis}

Statistical analyses were carried out using Statistical Package for Social Sciences (SPSS). The proportional data of this cross sectional study were tested using Pearson's Chi Square analysis test and Fisher Exact test.

\section{Results and Discussion}

In this study among the 175 Acinetobacter isolates the distribution of Acinetobacter species in various clinical specimens was in the following order, urine specimen 45 (25.71\%), endotracheal aspirate $41(23.43 \%)$, wound swab 41 (23.43\%), blood $17(9.71 \%)$, sputum (6.86\%), bronchial wash (4.00\%), PD fluid $(2.86 \%)$, CSF $(1.71 \%)$, pleural fluid $(1.14 \%)$ and ascitic fluid $(1.14 \%)$. The maximum number of Acinetobacter isolates were from respiratory samples $63(36.00 \%)$.

In the present study, four species of Acinetobacter were identified, of which A.baumannii $142(81.14 \%)$ the most common species followed by A.lwoffii 18(10.29\%), A.calcoaceticus 8 (4.57\%) and A.junii 7(4\%). There was a significant difference between the antimicrobial sensitivity pattern of A.baumannii and other species since $\mathrm{p}$ value is $<0.05$ for cephalosporins, aminoglycosides, quinolones and carbapenems. But there was no significant difference for cotrimoxazole and polymyxin-B. MDR was found to be in $60 \%$ of isolates. Of the 175 Acinetobacter isolates, 20 isolates (11.43\%) were found to be resistant to meropenem by Kirby Bauer disc 
diffusion method all of which belong to baumannii species. The MIC for the meropenem resistant isolates were shown in Table 2.

The phenotypic detection of resistant mechanisms showed that among the 20 isolates, MHT was positive in $9(45 \%)$ isolates, IEDT was positive in $9(45 \%)$ isolates, AmpC Disc test was positive in 6(30\%) isolates and $7(35 \%)$ isolates were negative for all the three phenotypic methods. Out of the 20 isolates, $3(15 \%)$ isolates were positive for MHT, IEDT and AmpC disc test and 7(35\%) isolates were positive for MHT and IEDT as shown in Table 3. On molecular detection all the 20 isolates were positive for OXA-23 $(100 \%), 9(45 \%)$ isolates were positive for blaVIM1 and 7 (35\%) isolates were positive for blaIMP1 which was shown in Table 4.

Finally comparison between phenotypic and genotypic methods for detection of meropenem resistance was done and the interpretation was shown in Table 5.

Acinetobacter infections present a global medical challenge because it is an important opportunistic GNB in health care institutions. It has gained importance because of its ability to survive under a wide range of environmental conditions, having numerous intrinsic and acquired drug resistance mechanisms and the emergence of multidrug and pandrug resistant strains. ${ }^{(17)}$ The isolation and identification of resistance pattern of Acinetobacter infections helps in selection of appropriate antibiotics, reducing the morbidity and mortality of the patients and in reducing the spread of resistant strains in the community.

A. baumannii $(81.14 \%)$ was the predominant species isolated in this study which is similar to the study conducted by Muktikesh Dash et al., where he documented that $79.6 \%$ isolates were A.baumannii, and Apoorva Tripathi et al., have reported that $74.50 \%$ isolates were A.baumannii. (18),(19) Similarly there was a significant difference ( $\mathrm{p}$ value $<0.05$ ) between the antimicrobial sensitivity pattern of A.baumannii and other species. In the present study, among the 20 meropenem resistant isolates, 17 isolates were resistant to imipenem and the remaining 3 isolates were sensitive to imipenem by disc diffusion. This is because of the difference in the pharmacodynamic property among the carbapenem drugs. ${ }^{(21)}$ This is similar to the study done by Muktikesh Dash et al., where $19 \%$ resistance to imipenem and $22 \%$ resistance to meropenem was documented. ${ }^{(18)}$

All the 20 meropenem resistant isolates have their MIC in the resistant range $(\geq 8 \mu \mathrm{g} / \mathrm{ml})$, hence disc diffusion method correlates with MIC (Table 2). This shows that the regular screening for meropenem resistance can be done using disc diffusion method as described in CLSI and further confirmed by MIC.

Among the 20 meropenem resistant isolates, Modified Hodge test was positive in 9/20 $(45 \%)$ isolates and Imipenem-EDTA combined disc test was positive in 9/20(45\%) isolates. This was similar to the study done by Gomty mahajan et al., (47.6\%). ${ }^{(6)}$ But MHT positivity varies between $2.2 \%$ to $71 \%$ in other studies. Similarly Gupta et al., Lee et al., Franklin et al., Sinha et al., and Uma et al., have documented $7.5 \%, 14 \%, 16 \%, 60.71 \%$ and $71 \%$ of MBL respectively. (5),(23),(24),(25) This may be due to lack of standardization of phenotypic procedures for detection of positive for both oxacillinase and metallo beta lactamase. This implies that combination of carbapenemases in NFGNBs, as there are no standard procedures described in CLSI and other similar guidelines and variations in the expression of MBL gene (Table 3). AmpC Disc test was positive in 6/20(30\%) isolates which was low when compared to Sinha et al., 
and Deepa et al., where they reported $60.71 \%$ and $73 \%$ of AmpC beta lactamases in carbapenem resistant isolates respectively. ${ }^{(11)}$ AmpC beta lactamases alone may not be the cause for carbapenem resistance but when it is present along with decreased membrane permeability, it is capable of conferring carbapenem resistance (Table 3 ). ${ }^{(20)}$

Among the 20 meropenem resistant isolates, $35 \%$ isolates were negative for all the three phenotypic methods, $15 \%$ isolates were positive for all the three tests and in $35 \%$ isolates several mechanisms exist in the same isolate to confer carbapenem resistance. ${ }^{(26)}$ Upon molecular characterization all the 20 isolates were positive for OXA-23 (100\%), 9 $(30 \%)$ isolates were positive for blaVIM1 and
$7(35 \%)$ isolates were positive for blaIMP1 (Table 4 and Fig 1, 2, 3). In this study, OXA23 was positive in all the 20 meropenem resistant isolates but MHT was positive only in 9 isolates. Similarly amongst $14 \mathrm{MBL}$ genes (blaVIM1 and IMP1) positive isolates, only 9 isolates tested positive in ImipenemEDTA combined disc test. Hence the detection of various carbapenemases by genotypic method was more sensitive when compared to phenotypic methods (Table 5). ${ }^{(27)}$

The clinical outcome of the patients with meropenem resistance was determined in this study. Among the 20 patients with meropenem resistant isolates, 5 patients expired when compared to $100 \%$ recovery in the meropenem susceptible group (155 patients).

Table.1 Primers: (Designed By Helini Biomolecules, Chennai)

\begin{tabular}{|c|c|c|}
\hline GENE & PRIMER SEQUENCES & AMPLICON SIZE \\
\hline \multirow{2}{*}{$\begin{array}{c}\text { OXA-23 } \\
\text { (F) } \\
\text { (R) }\end{array}$} & 5'-CTTGCTCGTGCTTCGACCGAGT-3' & \multirow[t]{2}{*}{$160 b p$} \\
\hline & 5'-CGCCTAGGGTCATGTCCTTTTC-3' & \\
\hline \multirow{2}{*}{$\begin{array}{c}\text { VIM1 } \\
\text { (F) } \\
(\mathrm{R})\end{array}$} & 5'-GTGCTTTGACAACGTTCGCT-3' & \multirow[t]{2}{*}{$422 \mathrm{bp}$} \\
\hline & 5'-TCCACGCACTTTCATGACGA-3' & \\
\hline \multirow{2}{*}{$\begin{array}{l}\text { IMP1 } \\
(\mathrm{F}) \\
(\mathrm{R})\end{array}$} & 5'-TTTTGCAGCATTGCTACCGC-3' & \multirow[t]{2}{*}{ 220bp } \\
\hline & 5'-CACGCTCCACAAACCAAGTG-3' & \\
\hline
\end{tabular}

Table.2 MIC for meropenem resistant isolates $(\mathrm{n}=20)$

\begin{tabular}{|l|l|l|l|l|l|l|l|l|l|l|l|l|}
\hline $\begin{array}{l}\text { MIC for } \\
\text { Meropenem }(\mu \mathrm{g} / \mathrm{ml})\end{array}$ & $\mathbf{5 1 2}$ & $\mathbf{2 5 6}$ & $\mathbf{1 2 8}$ & $\mathbf{6 4}$ & $\mathbf{3 2}$ & $\mathbf{1 6}$ & $\mathbf{8}$ & $\mathbf{4}$ & $\mathbf{2}$ & $\mathbf{1}$ & $\mathbf{0 . 5}$ & $\mathbf{0 . 2 5}$ \\
\hline $\begin{array}{l}\text { A. baumannii } \\
(n=20)\end{array}$ & - & 9 & 5 & 5 & 1 & - & - & - & - & - & - & - \\
\hline $\begin{array}{l}\text { P. aeruginosa } \\
\text { ATCC } \\
\text { 27853(control) }\end{array}$ & & & & & & & & & & & & \\
\hline
\end{tabular}

All the 20 isolates have their MIC values greater than $8 \mu \mathrm{g} / \mathrm{ml}$; hence they are resistant to meropenem. Among the 20 isolates, $9(45 \%)$ isolates have $256 \mu \mathrm{g} / \mathrm{ml}$ as MIC, $5(25 \%)$ isolates have $128 \mu \mathrm{g} / \mathrm{ml}$ as MIC, another $5(25 \%)$ isolates have $64 \mu \mathrm{g} / \mathrm{ml}$ as MIC and remaining 1(5\%) isolate has $32 \mu \mathrm{g} / \mathrm{ml}$ as MIC. 
Table.3 Phenotypic detection of resistant mechanisms of Acinetobacter species for meropenem $(n=20)$

\begin{tabular}{|c|c|c|c|}
\hline $\begin{array}{c}\text { PHENOTYPIC } \\
\text { TESTS }\end{array}$ & METHOD & $\begin{array}{c}\text { NO. OF } \\
\text { ISOLATES }\end{array}$ & $\begin{array}{c}\text { PERCENTAGE } \\
\%\end{array}$ \\
\hline \multirow{5}{*}{ POSITIVE } & $\begin{array}{l}\text { Modified Hodge Test } \\
\text { (MHT) }\end{array}$ & 9 & $45 \%$ \\
\hline & $\begin{array}{l}\text { Imipenem-EDTA combined disc } \\
\text { test (IEDT) }\end{array}$ & 9 & $45 \%$ \\
\hline & AmpC Disc Test & 6 & $30 \%$ \\
\hline & MHT, IEDT and AmpC Disc Test & 3 & $15 \%$ \\
\hline & MHT and IEDT & 7 & $35 \%$ \\
\hline NEGATIVE & MHT, IEDT and AmpC Disc Test & 7 & $35 \%$ \\
\hline
\end{tabular}

Table.4 Molecular detection of resistant mechanisms of Acinetobacter species for meropenem $(n=20)$

\begin{tabular}{|l|c|c|}
\hline \multicolumn{1}{|c|}{ GENES TESTED } & $\begin{array}{c}\text { NO OF ISOLATES WITH } \\
\text { GENES DETECTED }\end{array}$ & PERCENTAGE\% \\
\hline OXA -23 & 20 & $100 \%$ \\
\hline blaVIM1 & 9 & $45 \%$ \\
\hline blaIMP1 & 7 & $35 \%$ \\
\hline
\end{tabular}

Table.5 Comparison of phenotypic and genotypic methods in meropenem resistant isolates

\begin{tabular}{|c|c|c|c|c|c|c|}
\hline \multirow{3}{*}{$\begin{array}{c}\text { PHENOTYPIC } \\
\text { TEST } \\
\text { RESULTS }\end{array}$} & \multicolumn{6}{|c|}{ GENES TESTED } \\
\hline & \multicolumn{2}{|c|}{ OXA - 23} & \multicolumn{2}{|c|}{ blaVIM1 } & \multicolumn{2}{|c|}{ blaIMP1 } \\
\hline & Positive & Negative & Positive & Negative & Positive & Negative \\
\hline $\begin{array}{l}\text { Positive } \\
\text { MHT }(\mathrm{n}=9)\end{array}$ & 9 & - & 2 & 7 & 5 & 4 \\
\hline $\begin{array}{l}\text { Positive } \\
\text { IEDT }(n=9)\end{array}$ & 9 & - & 3 & 6 & 6 & 3 \\
\hline $\begin{array}{l}\text { Positive } \\
\text { AmpC Disc Test }(n=6)\end{array}$ & 6 & - & 2 & 4 & 3 & 3 \\
\hline $\begin{array}{l}\text { MHT, IEDT and } \\
\text { AmpC Disc test } \\
\text { Positive }(\mathrm{n}=3)\end{array}$ & 3 & - & 2 & 1 & 3 & - \\
\hline $\begin{array}{l}\text { Phenotypic tests } \\
\text { Negative }(n=7)\end{array}$ & 7 & - & 3 & 4 & 1 & 6 \\
\hline
\end{tabular}


Fig.1 PCR for OXA - 23 GENE

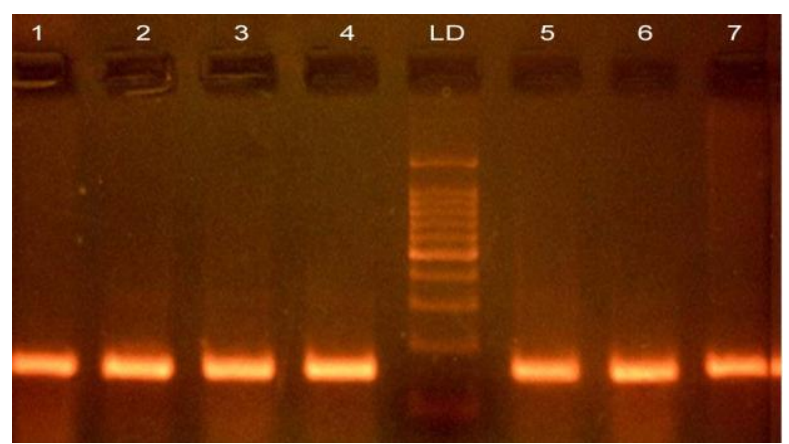

ISOLATE NO. - 1,2,3,4,5,6,7 (ALL POSITIVE FOR OXA - 23 GENE) LD- DNA LADDER

Fig.2 PCR for BLA - VIM1 GENE

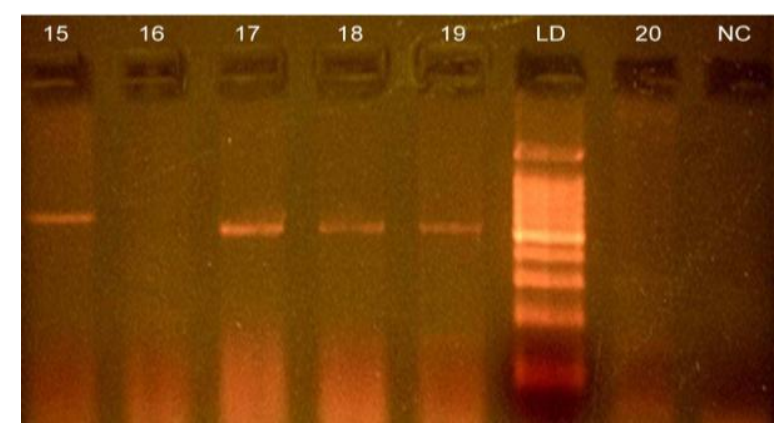

ISOLATE NO. - 15, 17, 18, 19 (POSITIVE FOR BLA - V1M1 GENE) LD- DNA LADDER, NC - NEGATIVE CONTROL

Fig.3 PCR for BLA - IMP1 GENE

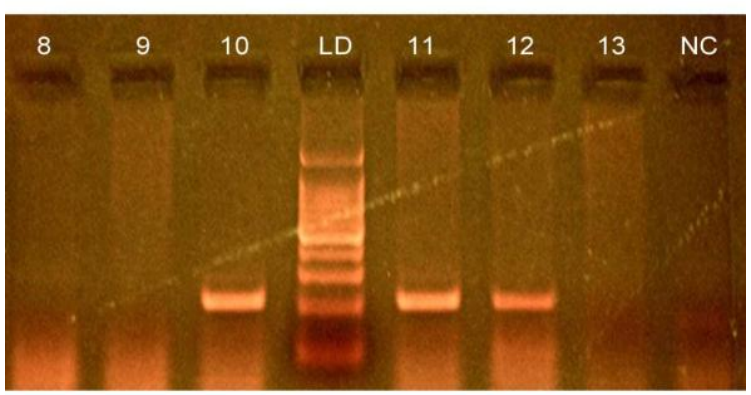

ISOLATE NO. - 10, 11, 12 (POSITIVE FOR BLA - IMP1 GENE) LD - DNA LADDER, NC NEGATIVE CONTROL

OXA-23 was positive in all the five patients, blaIMP1 was positive in 3 patients and blaVIM1 was positive in one patient.

All the three genes were positive in one patient. The mortality rate in patients with MBL gene was $21.43 \%$.
In conclusion, Acinetobacter species are the second most common nonfermenter isolated from clinical specimens next to Pseudomonas species. The infections caused by MDR Acinetobacter that are capable of producing various beta lactamases are associated with significant morbidity and mortality. Hence 
Acinetobacter has been added to the list of significant microbial challenges of current era.

Carbapenems remain the drug of choice for the MDR Acinetobacter infections. But resistance to carbapenems due to production of various beta lactamases is of great concern as they are encoded by genes which are horizontally transmissible. Though there is difference between phenotypic and genotypic methods in the sensitivity of detection of carbapenamases, genotypic methods remain the gold standard with more sensitivity and phenotypic methods due to ease of performance and cost effectiveness still remain useful for characterization of carbapenemase resistance.

The high prevalence of Acinetobacter infections emphasizes the need for early detection of various beta lactamases, which would help in selection of appropriate antibiotic regimen and prevention of emergence and dissemination of MDR strains. Hence, methods for detection of carbapenamases have to be standardized by formulating guidelines and be routinely used.

The present need is that all the health care institutions should have a coordinated effort to curtail inappropriate use of antibiotics, their own antimicrobial stewardship program, vigilant detection of resistant Acinetobacters, regular surveillance and infection control protocols to control the increasing incidence of highly resistant Acinetobacters.

\section{References}

1. Fellpe Fernandez, Luis Martinez, M Carman, Juan A. Relationship Between Betalactamase production, Outer membrane proteins and Penicillin binding proteins profiles on the activity of Carbapenems against clinical isolates of $A$. baumannii. J. Antimicrob. Chemo. 2003; 51(3): 565-74.

2. Loveena Oberoi, Nachhatarjit Singh, Poonam Sharma, Aruna Agarwal. ESBL, AmpC and MBL beta lactamases producing superbugs. Havoc in the ICU of Punjab, India. $\mathrm{J}$ of Clinical Diagnostic Reseach.2013; 7(1):70-73.

3. Rice LB. Challenges in Identifying New Antimicrobial Agents effective for treating infections with A.baumannii \& P.aeruginosa. Clin Infect Dis 2006; 43:100-5.

4. F Simsek, H Gedik, M T Yildirmak, NE Iris. Colistin against colistin-only susceptible A.baumannii related infections. Monotherapy or Combination therapy? Indian J Med Microbiol 2012; 30(14): 448-452.

5. M Sinha, H Srinivasa. Mechanisms of resistance to carbapenems in meropenem resistant Acinetobacter isolates from clinical samples. Indian $\mathbf{J}$ Med Microbiol. 2007; 25(2): 121-5.

6. Gomathy Mahajan, Sheevani Sheemar, Shashi Chopra et al., Carbapenem Resistance and Phenotypic Detection of Carbapenamases in Clinical isolates of A.baumannii. Indian $\mathrm{J}$ of Med sciences 2011; 65(1):18-25.

7. Kumar AV, Pillai VS, Dinesh KR, Karim S. The phenotypic detection of carbapenamase in meropenem resistant Acinetobacter calcoaceticus-baumannii complex in a $\mathrm{TCH}$ in South India. J Clin Diagn Res 2011; 5: 223-6.

8. Noyal MJ, Menezes GA, Harish BN, Sujatha S, Parija SC. Simple Screening tests for detection of carbapenamases in clinical isolates of nonfermentative gram-negative bacteria. Indian $\mathrm{J}$ Med Res 2009; 129: 707-12.

9. Lee K, lim YS, Yong D, Yum JH, Chong Y. Evaluation of the Hodge test and the imipenem EDTA DDST for differentiating MBL producing isolates 
of Pseudomonas aeruginosa \& Acinetobacter spp. J of Clin Microbiol 2003; 4623-9.

10. Anil VK, Vishnu SP, Kavitha R, Dinesh, Shamsul. The Phenotypic Detection of Carbapenamases in the Meropenem resistant A.calcoaceticus baumannii complex in a Tertiary care Hospital in a south India. $\mathbf{J}$ of Clin Diagnostic Research 2011; 5: 22-26.

11. Dheepa Muthusamy and Appalraju Boppe. Phenotypic Methods for the detection of various Betalactamases in Carbapenamase resistant isolates of A.baumannii in a Tertiary care Hospital in a south India. India. $\mathbf{J}$ of Clin Diagnostic Research. 2012; 6(6): 97073.

12. Yong D, Lee K, Yum JH, Shin HB, Rossolini GM, Chong Y. ImipenemEDTA disk method for differentiation of metallo- $\beta$-lactamases producing clinical isolates of Pseudomonas spp and Acinetobacter spp. J Clin Microbiol 2002; 40(3)798-801.

13. Black JA, Moland ES, Thomson KS. AmpC Disc test for detection of plasmid mediated ampC beta lactamase in Enterobacteriaceae lacking chromosomal ampC beta lactamases. J Clin Microbiol 2005; 43(3): 3110-3.

14. Coudron PE. Inhibitor based Methods for detection of Plasmid-mediated AmpC beta lactamases in Klebsiella spp.,Escherichia coli and Proteus mirabilis. J Clin Microbiol 2005;43:4163-7.

15. Ellington MJ, Kristler J, Livermore DM, Wood ford N. Multiplex PCR for rapid detection of genes encoding acquired metallo beta lacatamases. J Antimicrob Chemother 2007; 59:321-2.

16. Mastachio AK, Van der Heidjen I, Rossi F, Levin AS, Costa. Multiplex PCR for rapid detection of genes encoding oxacillinase and metallo beta lactamases in carbapenem resistant Acinetobacter species. J Med Microbiol 2009; 58:1522-4.

17. M Purohit, DK Mendiratta, VS Deotale, M Madhan et el. Detection of MetalloBeta lactamases producing Acinetobacter baumannii using microbiological assay, disc stnergy test and PCR. Indian $\mathrm{J}$ of Med Microbiol 2012; 30(4):456-61. 18. Muktikesh Dash, Sanghamitra Padhi, Swetlana Pattnaik, Indrani Mohanty, Pooja Misra. Frequency, risk factors, and antibiogram of Acinetobacter species isolated from various clinical samples in a tertiary care hospital in Odisha, India. Avicenna journal of Medicine 2013; 3(4):97-102.

19. Namita Jaggi, Pushpa Sissodia, Lalit Sharma. Acinetobacter baumannii isolates in a tertiary care hospital: Antimicrobial resistance and clinical significance. Journal of Microbiology \& Infectious Diseases 2012; 2(2):7-63.

20. Sinha N, Agarwal J, Srivastava S, Singh M.Analysis of Carbapenem-resistant Acinetobacter from a tertiary care setting in North India. Indian $\mathbf{J}$ Med Microbiol. 2013; 31:60-3.

21. Joseph L Kuti et al., Pharmacodynamics of Meropenem \& Imipenem against Enterobacteriaceae, A.baumannii \& P.aeruginosa. Pharmacotherapy 2004; 24(1).

22. CLSI document M100-S 24.vol.34 No.1 Tab-2B-2, Performance standards for Antimicrobial Susceptibility Testing; 24 informational supplements.Jan14, Interpretive standards for Acinetobacter spp.

23. Karthika RU, Rao RS, Sahoo S, Shashikala P, Kanungo R, Jayachandran S, Prashanth K. Phenotypic and genotypic assays for detecting the prevalence of metallo- $\beta$-lactamases in clinical isolates of Acinetobacter baumannii from a south Indian tertiary 
care hospital. J Med Microbiol 2009; 58: 430-435.

24. Lee K, lee WG, Uh Y, Ha GV, Cho J, Chong Y. VIM and IMP type MBL producing Pseudomonas species and Acinetobacter spp. in Korean Hospitals. Emerg Infect Dis 2003; 9:868-71.

25. Gupta V, Datta P, Chander J. Prevalence of MBL producing Pseudomonas spp. and Acinetobacter spp. in tertiary care hospital in India. J infect Dis.2006; 52:311- 4
26. Vikas Manchanda, Sinha Sanchalta and sNP Singh. Multi Drug Resistant Acinetobacter. J Glob Infect Dis 2010; 2(3):291-304.

27. Aleksandra, Agnieszka, Tomasz and Euginea. Usefulness of phenotypic and genotypic methods for metallo-betalactamases detection in carbapenemresistant Acinetobacter baumannii strains. Med Sci Monit Basic Res. 2013; 19: $32-36$.

\section{How to cite this article:}

Thiriveni, J., U. Umadevi and Lakshmipriya, N. 2018. Phenotypic and Molecular Characterization of Carbapenamase among Acinetobacter Isolates in a Tertiary Care Hospital, Tamil Nadu, India. Int.J.Curr.Microbiol.App.Sci. 7(12): 2762-2771. doi: https://doi.org/10.20546/ijcmas.2018.712.313 